MARÍA

GARCÍA ALCAIDE

Universidad de Alcalá de Henares

margarcialcaide@gmail.com

\title{
Anónimo era una mujer
}


ANONYMUS WAS A WOMAN

ABSTRACT

"Anonymous was a woman," wrote Virginia Woolf in her book A Room of one's own. We recover her quote with the aim of understanding how literature has always been linked to men, while woman remained in the background throughout history. Women writers signed under pseudonyms, hiding their names out of fear, shame or social pressure; some of them allowed the intrusion of their husbands, who gave themselves credit for their works, and others simply fell into oblivion. It is necessary to recover their memory in order to give them the place they deserve, especially in the educational field, where textbooks are full of male literature through which students learn to love, feel or cry as Lorca, Neruda or Benedetti did, thus annihilating a feminist vision at all levels where progress means also knowing how women love, feel or cry.

\section{Keywords}

Gender; feminism; education; literature.

\section{RESUMEN}

"Anónimo era una mujer", escribió Virginia Woolf en su libro Una habitación propia. Recuperamos su cita con el objetivo de comprender cómo la literatura ha estado siempre ligada al hombre, mientras que la mujer permanecía en un plano invisible a lo largo de la Historia. Las escritoras firmaban bajo pseudónimo, escondiendo sus nombres por miedo, vergüenza o presión social; algunas permitían el intrusismo de sus maridos, quienes se otorgaban los méritos de sus obras, y otras simplemente caían en el olvido. Es necesario recuperar la memoria para darles el lugar que se merecen, especialmente en el ámbito educativo, donde los libros de texto están repletos de literatura masculina mediante la cual el alumnado aprende a amar, sentir o llorar como lo hacían Lorca, Neruda o Benedetti, aniquilando así una visión feminista en todos los planos en los que progresar signifique también conocer cómo aman, sienten o lloran las mujeres.

\section{Palabras clave}

Género; feminismo; educación; literatura. 


\section{INTRODUCCIÓN}

Desde que Safo, primera poetisa occidental, reivindicase la importancia de la mujer en el mundo, han pasado más de tres milenios (López, 1997); casi tres mil años en los que las mujeres se han visto obligadas a recorrer un largo camino hasta una actualidad que, a pesar de haber subsanado algunos aspectos de la brecha de género y la desigualdad, todavía continúa estancada.

Así lo afirmó Virginia Woolf en su libro Una habitación propia, cuando escribió que "anónimo era una mujer", para reivindicar las dificultades con las que se han encontrado las mujeres a lo largo de la Historia para poder expresarse artísticamente. Recuperamos esta cita para reivindicar su presencia en la esfera social y educativa, ya que conocemos libros de gran envergadura, que se enseñan actualmente en las aulas, como El Cantar del Mio Cid (1200) que son anónimos y que, a pesar de su temprana edad de publicación y de la escasa presencia que tenía la mujer en la sociedad, podría haber sido escrito por una autora.

No obstante, con anónimo queremos ir más allá de la ausencia del nombre de las escritoras en una determinada publicación. Buscamos hacer un repaso histórico de todas aquellas mujeres que por miedo, vergüenza o presión social se vieron obligadas a escribir sin mostrar su identidad, ya fuese sin firmar su propio libro, escribiendo bajo pseudónimos masculinos, o escribiendo para sus maridos, convirtiéndose ellos en grandes intelectuales en el mundo de la literatura, mientras que ellas caían en el olvido.

Para ello, abordaremos en primer lugar un enfoque cronológico de las diferentes olas feministas, que nos sirva para entender la situación de la mujer en cada época y su evolución en la toma de conciencia de ella misma. Esto nos permitirá, más adelante, abordar diferentes apartados centrados en su desconocimiento como autoras (es decir, a través del movimiento feminista conoceremos cómo fue evolucionando su presencia como escritoras), el reconocimiento que se le ha otorgado a través de los diferentes premios y menciones literarias, y un presente que, aunque se plantea optimista, juega malas pasadas a las escritoras en el mundo editorial y literario.

Antes de continuar, debemos señalar que esta investigación se lleva a cabo desde un enfoque eurocentrista, donde analizamos la conexión y proximidad entre los impedimentos que encontraban las escritoras para ejercer su profesión, independientemente del país europeo que se tratase. Partir de esta perspectiva, nos lleva a ser conscientes de dos aspectos: en primer lugar, que la investigación aborda a mujeres blancas de la sociedad occidental y, en segundo lugar, reconocer que, aunque en este artículo no se aborde, el papel de las mujeres de otras zonas del mundo, como Asia o Latinoamérica, ha sido primordial en el desarrollo del movimiento feminista y de la escritura. Concluimos por tanto que, la reducción de este artículo a Europa es solo por cuestión de concreción y de proximidad. 


\section{CONTEXTO HISTÓRICO}

Esta investigación parte de un enfoque cronológico e histórico que nos permite investigar sobre cómo el desequilibrio en la igualdad de derechos es una constante que ha permanecido intacta desde los primeros movimientos feministas. Resultaría imposible realizar un estudio sobre las dificultades que tienen y han tenido las escritoras en el mundo literario, sin abordar su background histórico y contextual.

La lucha por las mujeres desde diferentes esferas sociales, políticas e ideológicas empieza a tomar forma a partir de la primera ola feminista, que comienza durante el desarrollo de El Siglo de las Luces (mitad del siglo XVIII), hasta la mitad del siglo XIX aproximadamente. En ese momento resaltan intelectuales como Olympie de Gouges (autora de la Declaración de los Derechos de la Mujer y de la Ciudadana escrito en 1791), que se enfrentan a autores como Rousseau, quien define a las mujeres como inferiores moralmente a los hombres, en su libro EI Emilio o De la Educación (García, 2002):

Toda la educación de las mujeres debe ser relativa a los hombres. Complacerles, serles útiles, hacerse amar y honrar de ellos, educarlos de jóvenes, cuidarlos de mayores, aconsejarles, consolarles, hacerles la vida agradable y dulce: he aquí los deberes de las mujeres en todos los tiempos y los que se les debe enseñar desde su infancia. (Rousseau, 1998, p. 417)

Lo mismo ocurrirá con otras pensadoras como Mary Wollstonecraft (autora de Vindicación de los derechos de la mujer, publicado en 1792), que se cuestiona los ideales de la Revolución Francesa, que pedían libertad, igualdad y fraternidad para todos, entendiendo como un todo a los hombres y quedando, por consiguiente, las mujeres excluidas de este colectivo. Este desacato constante a la sociedad del momento, le otorga a Wollstonecraft el apodo de "la hiena con faldas" (Varela, 2019, p. 43).

Afirma Nuria Varela en su libro Feminismo para principiantes, que el feminismo "nació siendo teoría y práctica" (2019, p. 35). Es decir, su evolución dio lugar desde un primer momento a libros que sientan las bases de este movimiento de forma teórica, pero que además nos permiten entender su ejercicio práctico a través de su participación activa en la promoción de lugares de pensamiento y culto, donde tomaban conciencia de su posición.

Que las mujeres han escrito desde siempre es una evidencia. Sus obras se han ido adaptando a la reivindicación política y social que vivían desde dentro hacia fuera, y aunque encontramos numerosas novelas firmadas con pseudónimos masculinos, de forma anónima o por otros hombres que se apropiaban de la obra, el papel de la mujer en la escritura aborda un amplio espectro que incluye todos los géneros y estilos, como también ensayos, panfletos políticos o discursos.

Se trata de un movimiento camaleónico, que ha ido pidiendo derechos según los cambios a los que la sociedad se enfrentaba. Así ocurrió durante la segunda ola (desde 1850 hasta 1950 aproximadamente), protagonizada por las sufragistas. En primer lugar, las sufragistas norteamericanas luchaban por un primer tema, que les llevo seguidamente a hablar de feminismo: abolir la esclavitud. Los esfuerzos realizados para conseguir su abolición les dio experiencia en la lucha, en la forma de expresarse y de defender sus ideas. 
Además, durante esta segunda ola, en Estados Unidos se estaba implantando la Reforma protestante que Lutero llevó a cabo en Europa en el siglo XVI. Se pretendía reestructurar los valores religiosos mediante una reforma moral que se enfrentase a las ideas de la Iglesia católica, y defendiese la interpretación libre y personal de las sagradas escrituras. Las doctrinas protestantes (Rossi, 1973) como las cuáqueras o evangelistas permitían que las mujeres tuviesen un papel activo en las oraciones y sermones. De esta forma, con una libre interpretación de las escrituras que ampliaba el abanico de quienes interpretaban, y un mayor acceso de las mujeres a la religión, el analfabetismo en Estados Unidos descendió y se dio paso a mujeres preparadas, como Lucretia Mott y Elizabeth Candy Stanton. Esta última fue propulsora de la Convención de Seneca Falls: foro de debate de los derechos de la mujer, desde donde surge la Declaración de Seneca Falls o Declaración de Sentimientos que busca favorecer los derechos políticos, económicos y sociales de las mujeres, y que se consolida como uno de los primeros programas políticos feministas (Varela, 2019).

Al hablar de la segunda ola, tenemos que detenernos en las sufragistas inglesas, que, tras varios intentos fallidos por proclamar su derecho al voto, y teniendo como aliados a John Stuart Mill y Jacob Bright, decidieron actuar a través de la interrupción de mítines políticos donde ofrecían sus propuestas de derecho al voto de las mujeres. Fueron encarceladas como presas comunes, no políticas (Varela, 2019) y una vez dentro, empezaron las huelgas de hambre. De esta época, destacan activistas como Lady Pankhurst, encarcelada en más de una ocasión, y a quien el rey Jorge $V$ encargó el papel de reclutar a las mujeres para que cubriesen los puestos laborales de los hombres durante la I Guerra Mundial.

Surgían por doquier argumentos en contra de las reivindicaciones feministas, de la mano de pensadores como Nietzsche, Schopenhauer, Hegel o Kierkegaard (De la Guardia, 2007) quienes defendían la reducción de las mujeres a objeto: mujer - madre; mujer - princesa, mujer - esposa y que, por consiguiente, influirían también en las escritoras.

Aunque esta investigación pretende centrarse en los obstáculos de las mujeres en la escritura, no podemos olvidar pasar por alto la relación del feminismo y el socialismo ya que, el movimiento feminista se ha visto siempre supeditado a los cambios que acontecían en la sociedad. Con el nacimiento del marxismo, o la aparición de El manifiesto comunista (escrito y publicado por Marx y Engels en 1848), parece establecerse una conexión inicial entre feminismo y marxismo, porque este último aborda un tema que era fundamental para el primero: las relaciones humanas entendidas como dominación y subordinación (Varela, 2019), pero en términos de revolución del proletariado, y no de dependencia de la mujer al hombre. Aunque hubo intentos por parte de pensadores como Engels de abordar esta cuestión, fue Clara Zetkin, directora de la revista femenina Igualdad, quien sentó las bases de un socialismo que luchase por las mujeres y sus derechos (Zetkin, 1976).

La lista de mujeres que tomaron parte en esta segunda ola se extiende a Emma Goldan, Alejandra Kollontai, Flora Tristán, entre otras. No podemos terminar el listado sin hablar de la escritora francesa Simone de Beauvoir y su libro El segundo sexo (1949), que creó una teoría sólida para el feminismo de todos los tiempos. La brillantez de Beauvoir fue cuestionada incluso por su padre, quien decía que su hija "tenía la inteligencia de un hombre" (Varela, 2019, p. 87). A lo largo de los dos volúmenes que tiene el libro, nos encontramos frente a una mujer que escribe sobre lo que supone serlo. Inicia con una cita de Pitágoras: "Hay un principio bueno que ha creado el orden, la luz y el hombre, y un principio malo que ha creado el caos, las tinieblas y la mujer" (De Beauvoir, 1949, p.11), y otra de Poullain de la Barre: "Todo cuanto han escrito 
los hombres sobre las mujeres debe ser sospechoso, pues son a un tiempo juez y parte" (De Beauvoir, 1949, p.11).

A finales de 1960 y principios de 1970 comienza la tercera ola feminista y, aunque existen opiniones distintas sobre cuándo terminó, va a ser la última que vamos a desarrollar en este apartado, ya que establecemos en esta investigación la cuarta ola como el momento actual en el que vivimos y del que analizaremos ejemplos prácticos más adelante. En este caso, se consideran que las cuestiones que se quieren conseguir son tan personales como políticas. Se defienden diferentes modelos de mujer, de género y de sexo y aparecen diferentes tipos de feminismos: el feminismo radical (destaca la obra de Kate Millet: Política sexual (1970) con jóvenes muy formadas académicamente, y que se interesaban por cuestiones como patriarcado, sexualidad y género bajo el lema: "lo personal es político", el feminismo cultural que desembocó en feminismo de la diferencia, el feminismo institucional desarrollado a raíz de las conferencias internacionales de la mujer, el feminismo académico que nace en las universidades, el ciberfeminismo que nace con motivo del desarrollo tecnológico o el ecofeminismo que centra su lucha también en la cuestión medioambiental.

Una vez hecho este repaso histórico sobre la lucha de las mujeres occidentales a lo largo de la Historia, solo nos queda recordar, antes de pasar al siguiente apartado, que conocer el pasado y contexto de todas las que nos precedieron nos sirve para entender los esfuerzos que se siguen haciendo en la actualidad. Es decir, siguiendo las palabras de Kate Millet en Política sexual: "el sexo es una categoría social impregnada de política" (Millet, 2010, p.68).

Como señalamos en la introducción, a lo largo de este recorrido histórico, nos hemos referido al movimiento feminista desde un enfoque generalmente occidental, y particularmente europeo. Es decir, a mujeres blancas de cierta posición económica y social. No obstante, esta investigación se hace siendo consciente de que el colectivo mujeres no es homogéneo, sino que engloba numerosas diferencias entre ellas, desde el color de piel, a su identidad de género, sexo, posición social, entre otros factores.

\section{MUJER, A TI QUE NADIE TE CONOCE}

Este apartado aborda ejemplos prácticos de aquellas personalidades femeninas que han sido silenciadas de una u otra forma. Resulta complicado hacer una lista cerrada, ya que el número de autoras que han sido calladas es interminable (Masiá, 2018). Seguiremos, no obstante, un orden cronológico que nos lleve a nuestros días.

Como ya sabemos, a mitad de siglo XVIII se produce la primera ola, con el nacimiento de un movimiento intelectual, conocido como Ilustración y la Revolución Francesa de 1789. Los grandes textos de la época como la Declaración de Derechos de Virginia sobre la independencia de Estados Unidos en 1776 o la Declaración de Derechos del Hombre y del Ciudadano en 1789 ignoraban la presencia de la mujer como parte de la ciudadanía. Por su parte, Rousseau aseguraba bajo la teoría del sujeto, basada en el racionalismo, que las mujeres no eran sujetos, sino complementos del hombre. Eran lo contrario a la razón, todo lo que estaba mal.

Ante estas declaraciones y una sociedad jerárquica de sexos con primacía de la esfera masculina, surge un feminismo ilustrado que pone en la mesa de debate la igualdad de derechos entre el hombre y la mujer, con exponentes como la francesa Olympie de Gouges, escritora, dramaturga 
y filósofa que escribió bajo un enfoque democrático igualitarista.

Tuvo una compañía de teatro itinerante a su mando y era una gran defensora de la abolición de la esclavitud, lo que pone de relieve que las mujeres razonaban sobre temas sociales trascendentales, que a muchos hombres les daban pánico por aquellos entonces (Varela, 2019, p.36). Con su obra La esclavitud negra (1792) fue encarcelada. Una vez puesta en libertad, y gracias a la Revolución, pudo ser interpretada en Comedie Franceise.

Sin duda, De Gouges debería de estar en los apuntes de secundaria sobre la Revolución Francesa. A día de hoy es uno de los máximos exponentes del feminismo. Su obra cumbre, la Declaración de los Derechos la Mujer y la Ciudadanía (1791), comienza diciendo: "Hombre, ¿̇eres capaz de ser justo? Una mujer te hace esta pregunta".

Destaca la carta que recibió de su padre, al conocer este la decisión de su hija por escribir:

No esperéis, señora, que me muestre de acuerdo con vos sobre este punto. Si las personas de vuestro sexo pretenden convertirse en razonables y profundas en sus obras, ¿en qué nos convertiríamos nosotros los hombres, hoy en día tan ligeros y superficiales? Adiós a la superioridad de la que nos sentimos tan orgullosos. Las mujeres dictarían las leyes. Esta revolución sería peligrosa. Así pues, deseo que las Damas no se pongan el birrete de Doctor y que conserven su frivolidad hasta en los escritos. En tanto que carezcan de sentido común serán adorables. Las mujeres sabias de Molière son modelos ridículos. Las que siguen sus pasos, son el azote de la sociedad. Las mujeres pueden escribir, pero conviene para la felicidad del mundo que no tengan pretensiones. (Citado en Blanco, 2000, p. 20)

Siguiendo con Mary Wollstonecraft, destacamos que después de ser institutriz durante un año, decidió abandonar su empleo en la casa para la cual trabajaba, para emprender su carrera como escritora (Varela, 2019, p.44). Fue muy criticada tanto política como personalmente por llevar una vida alejada de lo establecido como normal para aquella época y, no fue hasta inicios del siglo XIX, con la entrada en el panorama literario de nuevas escritoras feministas como Virginia Woolf que alaban su trabajo, cuando comienza a ser reconocida.

Es un caso curioso, porque a pesar de defender en su obra Vindicación de los derechos de la mujer (1792) que el motivo por el que las mujeres eran inferiores a los hombres eran por su inaccesibilidad a la educación, sostenía posteriormente que las mujeres aspiraban a ser hermosas, y que esta era su principal ambición (Wollstonecraft, 1792/1994). Esto ha originado posteriormente críticas sobre si era realmente feminista o no. No obstante, no podemos entender el feminismo de esta época como se entiende hoy en día.

Los ejemplos de estas pensadoras no eran lo común. Las mujeres solían acceder a la escritura por la vía religiosa, acercándose a un enfoque intimista y místico, que las alejaba de los nuevos conceptos revolucionarios y reformadores que llegaban con el siglo XVIII. Es el caso de Marcela de San Félix, la hija de Lope de Vega, o escritoras procedentes de la nobleza. Alejándose del concepto de escritora por vocación, estas lo hacían como una actividad intelectual más de su rutina diaria, que demostraba que la familia a la que pertenecía contaba con un buen nivel de cultura y educación.

En 1813, se publicó de forma anónima Orgullo y prejuicio, firmado como "Una novela en tres 
partes escrita por una dama". Fue escrito por Jane Austen y sin esperarlo, debido al éxito que tuvo, los lectores querían saber quién lo había escrito. Los miembros de su familia, como ejemplo de ese orgullo de pertenencia a una clase elevada, lo revelaron sin su consentimiento. Sin embargo, ella había cedido anteriormente los derechos de la novela, por lo que no obtuvo beneficios económicos (Álvarez, 2019). Entre las obras de Jane Austen, señalamos también Sentido y Sensibilidad (1811), Mansfield Park (1814) o Emma (1815).

A finales del siglo XVIII y principios del siglo XIX, a las mujeres se les permitía escribir acerca de los elogios anuales a la reina que formaban parte del ritual establecido para la entrega de premios de la Sociedad Económica de Amigos del País de Madrid y la Junta de Damas de Honor y Mérito. Es decir, en este contexto, la escritura en mujeres era vista como una actividad plebeya e indigna, salvo en aquellos casos en los que las mujeres abordaban temas institucionales y se dirigían solo a sus círculos más cercanos (Molina, 2015).

Las mujeres que deciden dedicarse a esta profesión a finales del siglo XVIII son llamadas "bachilleras" o "mujeres pedantes" (Fraile, 2004, p.13). Sin embargo, aquellas escritoras de Europa no eran todas iguales, ni vivían bajo las mismas circunstancias; ya que como hemos comentado anteriormente, no existe una homogeneización del colectivo mujer. La situación en la que se encontraban a finales de siglo determinaba su relación con los círculos intelectuales, el permiso de sus publicaciones, el contenido de sus escritos y su fama.

Con la entrada del Romanticismo, en el siglo XIX, se tenía la concepción de mujeres como seres dulces y sentimentales. De este modo, producirían literatura basada en emociones controladas y moderadas, que no tuviese nada que ver con lo que hacían los escritores románticos, más atrevidos. Gonzalo Morón en 1884, en su ensayo El destino de la mujer, explicó este argumento basándose en que las mujeres podrían escribir siempre y cuando no perdieran "el sentimiento del pudor y del recogimiento", y no despertasen "las peligrosas pasiones" (De la Guardia, 2000, p.6).

Durante la segunda ola feminista, las mujeres europeas reivindican especialmente el derecho al voto y el acceso a la educación, argumentos que sostenían pidiendo la igualdad de derechos entre hombres y mujeres (Aguilar, 2020). Recuperamos algunas citas del artículo "Las literatas. Carta a Eduarda" escrito por Rosalía de Castro en 1865, para entender la visión que se tenía de las escritoras desde fuera y su toma de conciencia acerca de cómo eran vistas:

Se dice que mi marido trabaja sin cesar para hacerme inmortal (...) todo es suyo. ¿Puede o escribir cosas que a ellos no se les han pasado nunca por las mentes, y eso que han estudiado y saben filosofía, leyes, retórica y poética? (...)Me ofende y lastima mi amor propio. (De Castro, 1865, p. 495).

Rosalía, a finales del Siglo XIX, nos está hablando de amor propio. Antes decíamos que las llamaban bachilleras, y así lo hace saber Rosalía de Castro en este artículo:

Si hablas de la tertulia y te expresas en un lenguaje correcto, te llaman bachillera, que lo quieres saber todo. Si vives apartada del trato de las gentes, es que te haces la interesante, estás loca. Las mujeres ponen de relieve hasta el más escondido de tus defectos y los hombres no cesan en decirte que una mujer de talento es una calamidad (...) Los hombres miran a las literatas peor que mirarían al diablo (...) Tu marido es el que escribe y tú la que firmas. (De Castro, 1865, p.494) 
Con su carta, Rosalía de Castro expresa presión, miedo, vergüenza, apropiación, ansias de libertad, rebeldía e incomprensión entre mujeres, o lo que hoy entenderíamos como falta de sororidad.

Las obras de escritores que encontramos a mitad del siglo XIX continúan con la "misoginia romántica" de Rousseau, que sitúa a la mujer como madre, esposa del sujeto, hogareña y religiosa. Las escritoras británicas y norteamericanas querían mostrar su enfado ante este perfil difundido de la mujer de la época a través de la búsqueda de su identidad: ¿Quiénes somos y cómo nos están representando los hombres? ¿Por qué no nos sentimos identificadas con ellas?

Las escritoras de este momento encontraron obstáculos a la hora de publicar sus obras y para obtener su reconocimiento tuvieron que hacerse pasar por hombres y utilizar pseudónimos. Es el caso de las hermanas Bronte: Charlotte, Emily y Anne. Emily es la autora de Cumbres Borrascosas (1847) y Charlotte de Jane Eyre (1847). Las tres hermanas firmaban respectivamente los libros bajo los pseudónimos de Currer, Ellis y Acton Bell (Costa, 2018).

Lo mismo ocurrió con la escritora Mary Evans, que firmó bajo el nombre de George Eliot la obra Middlemarch: un estudio de la vida de provincia (1871), considerada una de las mejores obras de la literatura inglesa (Costa, 2018). Resulta curioso su caso porque firmaba artículos con su verdadero nombre en el periódico, sin embargo, para ser reconocida en el mundo de la ficción, lo hacía bajo el pseudónimo masculino. Además, para que su trabajo fuese tomado en serio, escribió la obra Novelas tontas de ciertas damas novelistas (1856), que criticaba las novelas escritas por mujeres, para diferenciarse de otras autoras.

El uso de pseudónimos hacía hincapié en la vergüenza por escribir siendo mujer, la presión social que suponía serlo, el encuentro de la identidad como mujer que no se siente definida por los cánones, la sensación de libertad a través de la escritura y la protección de su persona, para evitar todos los peligros que acarreaba el escribir, como ser considerada loca:

1. Presión social y vergüenza: Las que publicaban utilizando sus propios nombres sufrían críticas de todo tipo. En muchos casos, el miedo a esta presión social les hizo no querer exponerse públicamente porque su expresión social no se correspondía a la representación tradicional.

2. Encuentro de la identidad: debido al movimiento de las sufragistas, conocemos que las mujeres pedían liberación para salir de la jaula, del castillo, de la casa. Nos encontramos entonces con autoras que se visten de forma masculina, que tienen relaciones amorosas tachadas de inapropiadas por la época, que se mueven en círculos intelectuales masculinos, etcétera. El hecho de que muchas de ellas publicasen toda su vida bajo un pseudónimo nos hace plantearnos que, probablemente algunas de esas mujeres en esa búsqueda de la identidad se encontrasen a ellas mismas y se diesen cuenta de que querían ser hombres, actuar como hombres, vestir como hombres, o querían ser mujeres, actuar como mujeres, vestirse como mujeres, pero como unas mujeres diferentes, que no tenían representación en la calle. Estaban encontrando su identidad de género.

3. Sensación de libertad: Publicar bajo el nombre de un hombre les daba la libertad de hablar de lo que quisieran, como quisieran, sobre quién quisieran. No tenían que preocuparse por lo que era moralmente concebido como la buena mujer.

4. Protección de la vida personal: Ante las restricciones de lo que debían escribir o no, existen 
numerosos temas sobre sexo, liberación o empoderamiento, de los que no estaba bien visto hablar. Cuando lo hacían, utilizaban pseudónimos para preservar su identidad y poder vivir tranquilas.

Por otro lado, había escritoras que trabajaban no con sus maridos, sino para ellos. Es el caso de Zelda Fitgerald, casada con Scott Fitzgerald. Gracias a la novela que publicó en solitario Save Me the Waltz (1932), basada en su matrimonio, se empezó a analizar su implicación en los libros de Scott, a quien enfadó su publicación y dijo que había utilizado elementos que él pensaba utilizar en sus libros (Corral, 2019). Ella expresaba su desesperación por tener éxito en algo propio. Como si todo lo que había hecho le hubiese sido arrebatado. Así lo manifestaba Virginia Woolf en Una habitación propia: "Una mujer debe tener dinero y una habitación propia si desea escribir" (2008, p.6). Propia en el sentido de la propiedad, porque nunca habían tenido nada.

A finales del siglo XIX y principios del siglo XX el concepto de mujer pasó por diferentes fases, de mujer - ángel y mujer - loca a mujer fatal y mujer - nueva. Encontramos, además, otros términos como la mujer - flor, la mujer - naturaleza, vidente y bruja dominada por el instinto sexual. Nos centraremos en la mujer nueva, heredada del movimiento sufragista, que nos interesa porque hace referencia a la mujer escritora, artista, liberada, con pelo más corto, falda más corta, pero también a la mujer loca, porque solía relacionarse con la poesía, la música y el arte en general.

Entran en juego "las sinsombrero", las escritoras españolas de la Generación del 27, que nos servirán para entender las mujeres caídas en el olvido. Si pensamos en la Generación del 27 se nos viene a la cabeza un núcleo cerrado formado por hombres como Alberti, Lorca, Cernuda o Juan Ramón Jiménez. Sin embargo, ¿qué ocurre con María Zambrano, Rosa Chacel, Concepción Arenal, Clara Campoamor, Victoria Kent, María Lejárraga, Zenonbia Camprubí, Carlota O’Neill?

Se necesitaría una tesis doctoral para hablar de cada una de ellas a título individual. A rasgos generales, escribían bajo el género autobiográfico y feminista ya que, debido al contexto histórico en el que nos situamos, España en 1927, la Guerra Civil había llevado al exilio de numerosos artistas y en el caso de ellas, el exilio político y femenino (Bianco, 2018). En esta etapa se produjo el legado de textos autobiográficos más amplio nunca antes generado.

Parecido a lo ocurrido con Zelda Fitzgerald, pasó con María Lejárraga y Zenobia Camprubí. La primera, casada con Gregorio Martínez Sierra, escribió la mayor parte de la obra de su marido. Por su lado, Zenobia se casó con Juan Ramón Jiménez y entregó su vida personal y profesionalmente a él (De la Fuente, 2007).

En esta época destacamos también el uso de pseudónimos, en este caso por miedo. Es el caso de Carlota O’Neill Lamo, periodista y escritora republicana progresista que para no ser reconocida por el franquismo usó un pseudónimo, en este caso, de mujer.

Entrando ya en la tercera ola del feminismo, existen posiciones diferentes acerca de si actualmente estamos en una nueva ola o seguimos en la tercera. En esta investigación, vamos a tomar la actualidad como una cuarta ola, entendiendo así la tercera ola como la última mitad del siglo XX y principios del siglo XXI. Sin embargo, nos serviremos de ambas para entender la evolución del último siglo como un fenómeno donde "anónimo era una mujer" continúa en la actualidad.

En estos años de la tercera ola, la teoría feminista pone sobre el tablero temas y palabras que 
hasta ahora no se habían escuchado como: el patriarcado, el uso de anticonceptivos, el control de la natalidad, la liberación sexual, el divorcio y la participación en la vida pública. El feminismo se hace más complejo y quiere devolver a la mujer al sitio que le corresponde, comenzando a su vez en 1970 la emergencia teórica del Feminismo.

Empiezan a ser conscientes de su capacidad artística y de la reducción que de ellas está haciendo la sociedad como cuerpo y sexo. En 1971, Linda Nochlin escribió el artículo “¿Por qué no ha habido grandes mujeres artistas?", donde argumenta que este factor se debe a los impedimentos sociales, culturales e institucionales con los que se han topado (López Fernández, 1991). A partir de esta época las mujeres ya no se esconden, pero siguen contando con numerosas trabas y estereotipos acuñados que llegan a nuestros días. Sucedió con J.K. Rowling, la autora de Harry Potter, quien se vio obligada a firmar algunos de sus relatos como Robert Galbraith, al mismo tiempo que llegó a reconocer que, en sus comienzos, sus editores preferían que firmase con sus iniciales con el único fin de dar ambigüedad a su sexo.

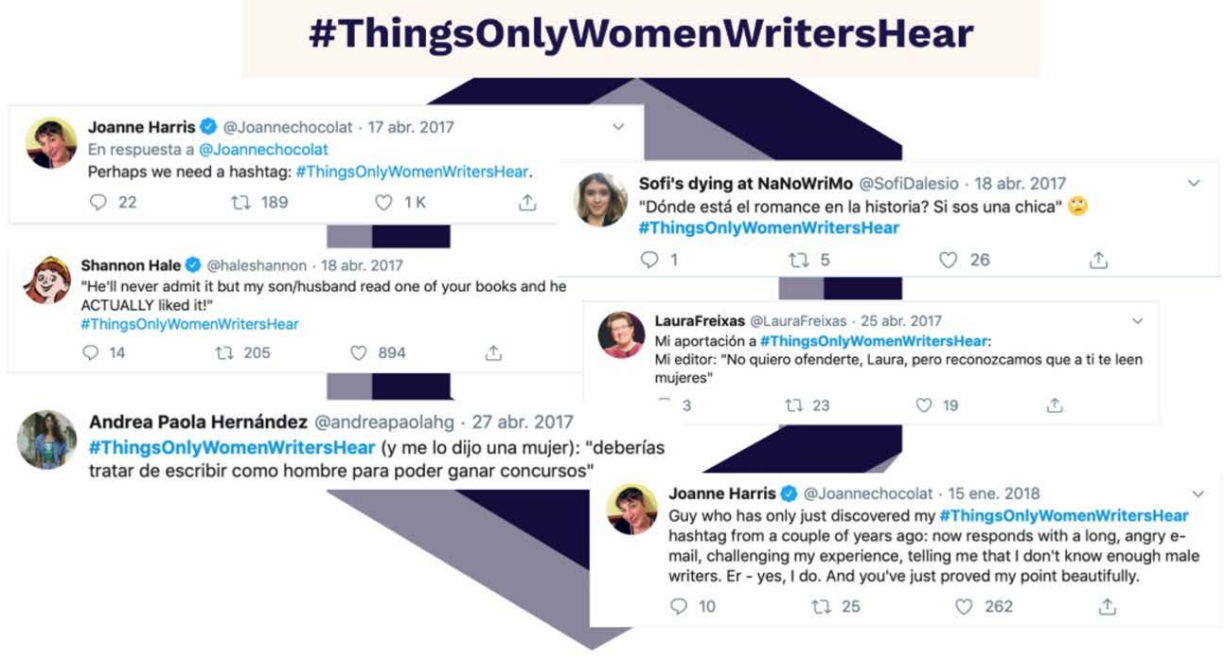

Figura 1.

Por otro lado, en 2017 surge el movimiento \#ThingsOnlyWomenWritersHear (Cosas que solo escuchan las escritoras), impulsado por la autora de Chocolat, Joanne Harris. A través de Twitter quiso visibilizar la realidad y micromachismos a los que se enfrentaban las autoras publicando un tweet con el hashtag arriba mencionado. Escritoras de todas partes del mundo se sintieron identificadas y se animaron a responder, contando sus historias. Algunos de estos tweets se recogen en la Figura 1. 
Tras una revisión bibliográfica y cronológica de la presencia femenina en la escritura occidental europeizada, llegamos al siglo XXI, donde el paradigma ha cambiado en cuanto a libertades. Sin embargo, no lo ha hecho en cuanto a reconocimiento y valor, por lo que el olvido sigue estando muy presente (De la Guardia, 2007). Además, en el sistema educativo el alumnado se ha nutrido de referencias varoniles en todos los ámbitos, mientras que no han recibido información e influencia de la posición que tenían las mujeres en el mundo académico y literario (López, 2014):

- Hasta la fecha solo 14 mujeres han ganado el Premio Nobel de Literatura desde su creación en 1901.

- El Premio Cervantes, reconocimiento de mayor envergadura en lengua castellana, únicamente ha galardonado a 4 mujeres frente a 38 hombres desde su creación en 1976: María Zambrano (1988), Dulce María Loynaz (1992), Ana María Matute (2010) y Elena Poniatowska (2013).

- El Premio Planeta, otro de los premios de alto rango en el país, ha distinguido a 16 mujeres desde 1952.

- La Real Academia de la Lengua Española parece ser un espacio reservado a la esfera masculina, siendo solamente once mujeres las "privilegiadas" de pasar por sus sillas. Carmen Conde, uno de los grandes nombres olvidados de la generación del 27, fue la primera elegida para ocupar el asiento K en 1979, quien fue sustituida, tras su muerte por Ana María Matute. En 1984, Elena Quiroga fue seleccionada para formar parte de esta institución. Tras ella, llegaron la historiadora Carmen Iglesias en 2002 y la bioquímica Margarita Salas en 2003.

En esta ventana al presente nos encontramos con un futuro prometedor, donde existen escritoras a nivel nacional como Elvira Sastre (Segovia, 1992), Sara Búho (La Línea, 1991) o Andrea Valbuena (Barcelona, 1992) que, por ejemplo, en poesía, están transformando la métrica. Son embajadoras del verso libre y hablan acerca de todo, poniendo en el ojo del huracán una gran cantidad de temas que sirven para visibilizar: el amor en todas sus formas, la bisexualidad, el colectivo LGTBIQ+, el aborto, el dolor, la soledad, el derecho a estar triste. Han llegado para contarnos su versión, y esa versión ha atravesado literalmente el charco, conectando con otras autoras como Fernanda Trías (Uruguay, 1976) o Rupi Kaur (India, 1992). Las escritoras millennials vienen pisando fuerte y, gracias a las redes sociales, y al buen uso que están haciendo de ellas, cada vez son más los jóvenes que encuentran referencias femeninas en la literatura.

Esto se debe a un cambio en el contexto sociocultural: se trata de mujeres que han nacido en democracia y en la era millennial, con un auge de las redes sociales que les ha dado mayor democratización y visibilidad a escala internacional (Pérez Alonso, 2015). Sin duda, nos permite coger aire fresco y pensar que, en un futuro optimista e igualitario, sus nombres aparecerán en los libros de texto.

También nacen nuevos proyectos creados y gestionados por mujeres, que buscan poner de relieve la sororidad en el mundo editorial y literario femenino y ayudar a dar visibilidad a todas las voces femeninas. Es el caso de editoriales independientes como LaCarmensita (ver Fig. 2) o Ediciones en el mar, editorial que se define en su cuenta de Instagram (@edicionesenelmar) como: "queremos hacer libros por los que nos hubieran quemado en la hoguera", y de proyectos 
como Las Rosas de Woolf (ver Fig. 3), un podcast literario con perspectiva de género que desarrolla en cada episodio el análisis de un libro desde un enfoque feminista.

PRODUCTS NOSOTRAS MANUSCRITOS CONTACT CART

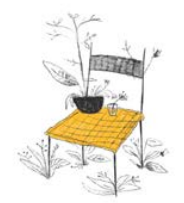

La Carmensita Editorial

\title{
MANUSCRITOS
}

\begin{abstract}
La Carmensita nace entre palmas, al solito del levante, cobijado por olor a mandarinas y salitre. La Carmensita se crea como un espacio donde sacar las sillas al patio y reunirte con tus vecinas para recitar, contary sacar lo que se lleva dentro. Aquí editaremos libros de poemas escritos por mujeres que buscan ese patio. Mimaremos y pondremos atención en cada proyecto para crear pequeñas piezas de esas a las que siempre volver. Como lo son los pueblos, como lo son la familia, como lo son las raíces. Volver. Volver a lo que, de algún modo, deposita un poco de calma en nosotras.
\end{abstract}

Figura 2.

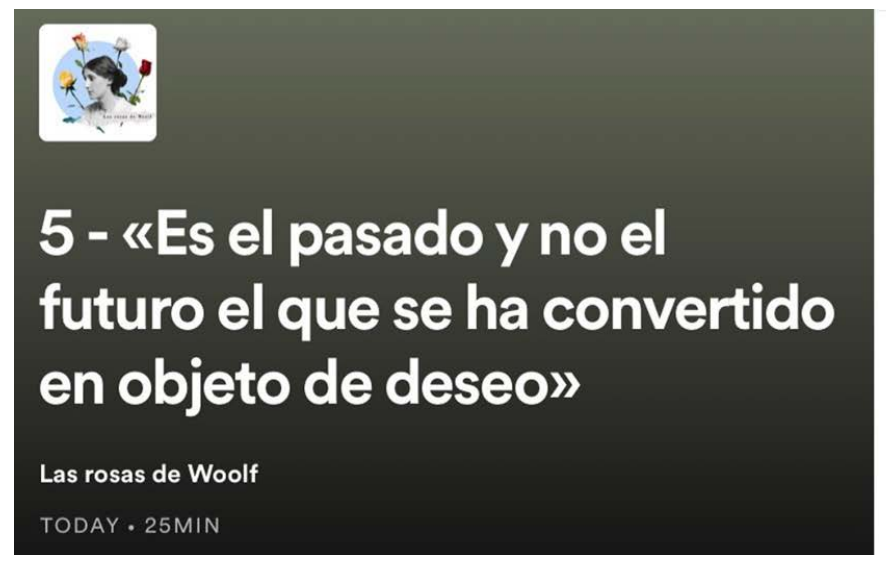
2. lasrosasdewoolf ¡Yaaaaaa! ¡Por fin tenemos nuevo episodio del podcast! Como os prometimos, en esta ocasión hablamos de «Los años», de Annie Ernaux, un libro que nos ha ENCANTADO y nos ha dejado con muchísimas ganas de seguir leyendo a esta autora. ¡Esperamos que os guste! ? \#podcast \#feminismo \#bookish \#bookstagram \#annieernaux $7 \mathrm{sem}$

Figura 3.

Existe, por tanto, ese techo de cristal y un intento efervescente y continuo para romper con su inaccesibilidad en términos de igualdad a la esfera literaria. 
Tras hacer un estudio sobre la presencia de las mujeres occidentales y europeas en la escritura, concretamos que la violencia machista se plasma históricamente en la negación de la capacidad artística de las mujeres por el miedo a que su educación llevase de la mano su revolución hacia el mismo nivel que los hombres.

En la actualidad, podemos decir que las escritoras han conseguido romper con el canon occidental y europeo que asocia mujer con romanticismo y feminidad, mostrando en sus obras los sentimientos de todas las formas posibles. Nos están enseñando a conocer el amor, el sexo, el dolor o la libertad en todas sus vertientes y formatos. Y a su vez, nos permiten conocer nuevas sensaciones que hasta ahora habían sido interpretadas por los hombres, como es el caso de la maternidad o la virginidad en la mujer.

Gracias a la aparición de las redes sociales se está destruyendo el silencio que mantenía las mujeres a la sombra. Con ellas, no solo se consigue que el alcance sea mayor, sino que también el público sea más diverso e inclusivo en sus propuestas literarias.

\section{REFERENCIAS}

Aguilar, N.. (2020). Una aproximación teórica a las olas del feminismo: la cuarta ola. Málaga. Universidad de Málaga.

Álvarez, I. (2019). Jane Austen, precursora del feminismo. Un estudio a través de su novela Orgullo y prejuicio. Madrid. Universidad Pontificia Comillas.

Bianco, S. (2018). Las Sinsombrero: mujeres olvidadas de la generación del 27. Las inéditas: voces femeninas más allá del silencio, (21-33). Universidad de Salamanca. Recuperado de: https://eusal.es/index.php/eusal/catalog/view/978-84-9012887-9/5222/5148-1

Blanco, O. (2000). Olympie de Gouges (1748 - 1793). Madrid: Ediciones del Orto.

Costa, C. (24 de noviembre de 2018). Las escritoras que tuvieron que usar pseudónimos masculinos y ahora serán leídas con sus nombres verdaderos. BBC News Brasil. Recuperado de: https:// www.bbc.com/mundo/noticias-46293652

De Beauvoir, S. (2012). El segundo sexo (4o ed.). México: Random House Mondadori, S.A.

De la Guardia, C. (2009). La violencia del nombre. Mujeres, seudónimos y silencios. En P. Pérez Cantó (Coord.), El origen histórico de la violencia contra las mujeres (pp. 201-240). Madrid: 
Dilema. Recuperado de: https://dialnet.unirioja.es/servlet/ articulo?codigo $=4073976$

De Castro, R. (1865). Las literatas. Carta a Eduarda. Almanaque de Galicia de Lugo, (493 - 495). Recuperado de: http://culturagalega. gal/album/docs/doc_16_literatas.pdf

De la Fuente, I. (2007). Zenobia Camprubí, amor y dependencia. Revista Clarín. Recuperado de: https://revistaclarin.com/421/ zenobia-camprubi-amor-y-dependencia/

Fraile, D. (2004). Mujer y cultura: la educación de las mujeres en la Edad Moderna. Foro de Educación, (4), 13. Recuperado de: http://dialnet.unirioja.es

García, R. (2002). Mujeres, arte y literatura: Imágenes de lo Femenino y Feminismo. Instituto de Investigaciones Feministas. Universidad Complutense de Madrid. Recuperado de: https:// www.ucm.es/data/cont/media/www/pag-44805/1CuadernosMujeres,\%20arte\%20y\%20literatura.pdf

Corral, E. (2019). Zelda Sayre: la flapper olvidada. Universidad Autónoma de Barcelona, Barcelona. Recuperado de: https:// ddd.uab.cat/pub/tfg/2019/tfg_178835/Zelda_Sayre_la_flapper_ olvidada_Esther_Corral_Gomez_-_TFG.pdf

Iborra, E.G. (2018). Una potente literatura escasa de premios. El correo. Recuperado de: https://www.elcorreo.com/culturas/ territorios/potente-literatura-escasa-20181013152041-nt.html

López, A. (1997). Safo como referente de las poetas hispanas del siglo XIX y XX. Revista De Estudios De Antigüedad Clásica, (8), 221-241. Recuperado de: https://dialnet.unirioja.es/servlet/ articulo?codigo $=165490$

López Fernández, M.A. (1991). ¿Por qué no ha habido grandes mujeres artistas? (Linda Nochlin, 1971). Comentario y recensión. Revista Arte, Individuo y Sociedad, 4, 205 - 211.

Masiá, C. (2018). Historia de las mujeres que hicieron historia. Madrid: Alba Libros.

Millet, K. (2010). Política sexual. Feminismos. Madrid: Ediciones Cátedra.

Molina, I. (2005). La ficción doméstica: Ángela Grassi, Pilar Sinués y Faustina Sáez. Una aproximación de género en la España burguesa. Universidad de Valencia, Valencia. Recuperado de: https://core.ac.uk/download/pdf/71057223.pdf 
Pérez Alonso, J.E. (2015). Narradoras en tránsito. Voces propias de escritoras nacidas en los 70. Dossiers Feministes, 20, 123139. Recuperado de: https://dialnet.unirioja.es/servlet/ articulo?codigo $=5440367$

Rossi, A.S. (1973). The Feminist Papers. Nueva York: Bantam Books. En Varela, N. (2019). Feminismo para principiantes.

Rousseau, J.J. (1998). Emilio o De la Educación. Madrid: Alianza Editorial.

Varela, N. (2019). Feminismo para principantes. (3o ed.) Barcelona: Penguin Random House.

Wollstonecraft, M. (1994). Vindicación de los Derechos de la Mujer. Recuperado de: https://teoriapoliticaseminariohome. files.wordpress.com/2020/02/vindicacic3b3n_de_los_ derechos_de_la_mujer.pdf

Woolf, V. (2008). Una habitación propia (3o ed.). Barcelona: Editorial Planeta.

Zetkin, C. (1976). La cuestión femenina y la lucha contra el reformismo. Barcelona: Anagrama. 